\title{
Infrared Observations of Interstellar Ices - Laboratory Needs
}

\author{
A.C.A. Boogert \\ California Institute of Technology, Downs Laboratory of Physics 320-47, \\ Pasadena, CA 91125, USA
}

\begin{abstract}
A selection of recent highlights in the area of infrared observations of interstellar ices is presented. The essential role that laboratory astrophysics plays in the interpretation of the data, and current problems and expected future needs are discussed.
\end{abstract}

\section{Introduction}

The idea that sticking of atoms and molecules to cold grains in dense clouds leads to the formation of icy mantles (Oort \& van de Hulst 1946), is now observationally well established. While much work has been done from the ground and airplanes (see Whittet 1992 for a review), the Infrared Space Observatory (ISO) has given us the first, full and unhindered view of the spectral region where the vibrational transitions of interstellar ices occur, 2-20 $\mu \mathrm{m}$. Approximately 30 broad absorption bands have been detected, attributed to $\sim 16$ different, mostly simple hydrogenated and oxidized molecules. New detections or necessary confirmations made in the last 5 years, include important molecules such as $\mathrm{CO}_{2}$ (Gerakines et al. 1999), $\mathrm{NH}_{3}$ (Lacy et al. 1998), and $\mathrm{CH}_{4}$ (Boogert et al. 1998). Detailed studies of the ice inventory were made toward high mass protostars (Gibb et al. 2000), low mass protostars (Boogert et al. 2000a), obscured field stars (Whittet et al. 1998), and the galactic center (Chiar et al. 2000).

The wealth of information contained in this data can however only be appreciated using the powerful technique of laboratory spectroscopy of analogs of interstellar ices. It allows us to answer a number of key questions on the basic physics and chemistry involved in the formation, evolution, and destruction of molecules in interstellar, circumstellar, and (proto)planetary space.

\section{Identification of Ice Bands and Band Profile Analysis}

As an illustration of the use of laboratory spectroscopy in the analysis of this data, we discuss here the ISO observations of the $4.38 \mu \mathrm{m}$ absorption band (Boogert et al. 2000b). The profile of this band varies significantly between a number of lines of sight, in particular toward high mass protostars. On top of a broader feature, there is an additional narrow structure present toward some lines of sight. Extensive laboratory studies (Ehrenfreund et al. 1999; Dartois et al. 1999), reveal that the broader component is reproduced by $\mathrm{CO}_{2}$ mixed with the polar molecules $\mathrm{H}_{2} \mathrm{O}$ and $\mathrm{CH}_{3} \mathrm{OH}$, while the narrow feature is a result of 
heating and crystallizing this ice. Indeed, sources with a more prominent narrow substructure, also have higher dust temperatures and gas/solid abundance ratios. It is thus concluded that thermal heating plays a major role in the ices around massive protostars. The $\mathrm{CO}_{2}$ ice bands may even trace the evolutionary stage of the object (Boogert et al. 2000b; van der Tak et al. 2000).

While the $\mathrm{CO}_{2}$ ice bands are securely identified (however, see Sect. 3), the identification of some bands needs further laboratory study. Examples are the weak 5.8, 7.25, and 7.4 $\mu \mathrm{m}$ bands (Schutte et al. 1998; Keane et al. 2000). The most famous example, however, is the $6.85 \mu \mathrm{m}$ band that is observed in almost all massive protostars. Although several identifications were proposed $\left(\mathrm{NH}_{4}^{+}\right.$, $\mathrm{CH}_{3} \mathrm{OH}$ ), at present none of these obey the strong constraints put by the ISO observations. Whatever the carrier is, the variation in band profile observed between many sources, suggests the important of thermal processing also for this band (Keane et al. 2000).

Although there is much evidence that thermal processing is an important process in the interstellar medium, the spectroscopic signatures of other physical or chemical processes are much weaker. The presence of the $4.62 \mu \mathrm{m}$ XCN absorption feature suggests however, that energetic processing (UV, cosmic rays) can not be neglected (Pendleton et al. 1999). For a discussion on laboratory work on energetic processing, we refer to Schutte (this volume).

\section{The Need for Optical Constants}

Sometimes neglected in the past, has been the importance of Mie scattering on absorption band profiles. Some abundant species with intrinsically narrow absorption bands, and large peak strengths can have very different absorption profiles, depending on the shape of the dust grain. For the likely case of a distribution of grain shapes along the line of sight, the net effect is a more prominent short wavelength shoulder and a general broadening of the profile. The astrophysically most relevant molecules in this case are the $\mathrm{CO}$ and ${ }^{12} \mathrm{CO}_{2}$ ice bands (Tielens et al. 1991; Ehrenfreund et al. 1997).

The unknown grain shape adds an extra degree of freedom to the analysis of interstellar ice bands. Accurate optical constants are essential. Unfortunately, the optical constants produced by various laboratory groups are not in agreement (Ehrenfreund et al. 1997). The effect of Mie scattering is almost negligible when the optical constants from one group are used (Trotta 1996), but very large when using another group's constants (Hudgins et al. 1993; Ehrenfreund et al. 1997). Recent polarization measurements of laboratory $\mathrm{CO}$ and $\mathrm{CO}_{2}$ ices, have provided some clarification in this dilemma (Baratta, Palumbo, \& Strazzulla 2000 ), in the sense that the effects of Mie scattering are not negligible, possibly in conflict with the results of Trotta (1996). We emphasize however, that further measurements of optical constants for a range of mixtures and temperatures are needed.

\section{Future Work}

Laboratory spectroscopy has been an essential tool in the interprctation of observations of interstellar ices. A continued combined observational-laboratory 
effort is required in the near future with the availability of sensitive high resolution spectrometers, with large infrared arrays (NIRSPEC, ISAAC, VISIR) on 8-10 meter class telescopes (Keck, VLT, Gemini). These instruments will provide detection of ice features toward a class of objects that could be studied only in a limited way until now, such as low mass protostars. With these observations it will become possible to make the very important link between interstellar and cometary ices. Concluding, infrared spectroscopy of interstellar ices is an active and promising research area, driven by the availability of excellent new observing facilities, as well as our proven ability to simulate these processes in laboratories.

\section{References}

Baratta, G.A., Palumbo, M.E., \& Strazzulla, G. 2000, A\&A, 357, 1045

Boogert, A.C.A., Helmich, F.P., van Dishoeck, E.F., Schutte, W.A., Tielens, A.G.G.M., \& Whittet, D.C.B. 1998, A\&A, 336, 352

Boogert, A.C.A., Tielens, A.G.G.M., Ceccarelli, C., et al. 2000a, A\&A, 360, 683 Boogert, A.C.A., Ehrenfreund, P., Gerakines, P.A., et al. 2000b, A\&A, 353, 349

Chiar, J.E., Tielens, A.G.G.M., Whittet, D.C.B., et al. 2000, ApJ, 537, 749

Dartois, E., Demyk, K., d'Hendecourt, L., \& Ehrenfreund, P. 1999, A\&A, 351, 1066

Ehrenfreund, P., Boogert, A.C.A., Gerakines, P.A., Tielens, A.G.G.M., \& van Dishoeck, E.F. 1997, A\&A, 328, 649

Ehrenfreund, P., Kerkhof, O., Schutte, W.A., et al. 1999, A\&A, 350, 240

Gerakines, P.A., Whittet, D.C.B., Ehrenfreund, P., et al. 1999, ApJ, 522, 357

Gibb, E.L., Whittet, D.C.B., Schutte, W.A., et al. 2000, ApJ, 536, 347

Hudgins, D.M., Sandford, S.A., Allamandola, L.J., \& Tielens, A.G.G.M. 1993, ApJS, 86, 713

Keane, J.V., Tielens, A.G.G.M., Boogert, A.C.A., Schutte, W.A., \& Whittet, D.C.B. 2000, A\&A, in press

Lacy, J.H., Faraji, H., Sandford, S.A., \& Allamandola, L.J. 1998, ApJ, 501, 105 Oort, J.H., \& van de Hulst, H.C. 1946, BAN, 10, 187

Pendleton, Y.J., Tielens, A.G.G.M., Tokunaga, A.T., \& Bernstein, M.P. 1999, ApJ, 513, 294

Schutte, W.A., Boogert, A.C.A., Tielens, A.G.G.M., et al. 1998, A\&A, 343, 966 Tielens, A.G.G.M., Tokunaga, A.T., Geballe, T.R., \& Baas, F. 1991, ApJ, 381, 181

Trotta, F. 1996, Thesis, LGGE-CNRS, Université Joseph Fourier, Grenoble van der Tak, F.F.S., van Dishoeck, E.F., Evans II, N.J., \& Blake, G.A. 2000, ApJ, 537, 283

Whittet, D.C.B. 1992, Dust in the galactic environment (IOP Publishing, Bristol)

Whittet, D.C.B., Gerakines, P.A., Tielens, A.G.G.M., et al. 1998, ApJ, 498, 159 